\title{
ANALYTICAL STUDY ON THERMOHALINE CONVECTIVE INSTABILITY IN A MICROPOLAR FERROFLUID
}

\author{
Nirmala P Ratchagar ${ }^{a}$, Seyalmurugan \\ ${ }^{\text {A, }}{ }^{a}$ Department of Mathematics, Annamalai University, Chidambaram, Tamilnadu - 608002 , India \\ ${ }^{b}$ Department of Mathematics, Jayagovind Harigopal Agarwal Agarsen College, Madhavaram, Chennai, Tamilnadu - 600060 , \\ India
}

Article History: Received: 11 January 2021; Accepted: 27 February 2021; Published online: 5 April 2021

\begin{abstract}
The present investigation is on linear analytical study of thermohaline convective instability in micropolar ferrofluid using perturbation technique. The fluid layer is heated from below and salted from above. The theory of linear stability is used to reduce the non-linear effects on governing equations and normal mode analysis is taken to study. The critical magnetic thermal Rayleigh number $N_{S C}$ is obtained for sufficient large value of $M_{1}$ and an oscillatory instability is determined. The parameters $N_{1}$ and $N_{5}{ }^{\prime}$ are analyzed for stabilizing behavior and $N_{3}{ }^{\prime}, \tau$ and $M_{3}$ give the destabilizing behavior. The results are depicted graphically.
\end{abstract}

Key words:

Thermohaline convection, Micropolar ferrofluid, Salinity Rayleigh number, Perturbation technique, linear stability analysis

\begin{abstract}
1. Introduction
Ferrofluids are colloidal suspension of fine magnetic mono domain nano-particles in non - conducting liquids. Such types of ferrofluids have wonderful applications in science and technology. Generally, the ferrofluids are used for cancer treatment in the biomedicine field. An excellent introduction and reviews of this extremely interesting monograph has been given by Rosensweig [1]. In his monograph, the fascinating information is introduced on magnetization. The convection in ferromagnetic fluid is analyzed in various aspects by Chandrasekhar [2]. Finlayson [3] has been investigated the convection in ferrofluid in single component fluid with uniform magnetic fluid. This investigation is extended to porous medium by Vaidyanathan et al. [4]. In nonpresence of buoyancy effects, the thermoconvective instability in ferrofluid is given by Lalas and Carmi [5].

The micropolar fluids respond to spin inertia and micro-rotational motions. It can support couple stress and distributed body couples. Eringen [6] introduced the micropolar fluids theory. This theory has been developed by Eringen [7] on thermal effect. An excellent reviews and applications of this fluids theory can be obtained in by Ariman et al. [8] and Eringen [9]. Later, Ahmadi [10] employed firstly the energy method on convective instability of micropolar fluid with use of stability analysis. Pérez-Garcia and Rubi [11] analyzed the micropolar fluids with the effects of overstable motions. Narasimma Murty [12] examined the instability of the Bénard convection in a micropolar fluid using linear stability analysis.

In the effect of porous media, the double-diffusive convection is of greatest interest in mechanical and chemical engineering. In some special case, sodium chloride and temperature field are involving and this is often called as thermohaline convection. Thermohaline convection in a ferrofluid has been analyzed by Vaidyanathan et al. [13] with two-component fluid. The presence of porous medium on ferrothermohaline convection has been given by Vaidyanathan et al. [14].

The theoretical investigation of a micropolar ferromagnetic two component fluid in non-presence of Darcy porous effect has been undertaken by Sunil et al. [15]. The Soret effect is investigated on two component ferrofluid by Vaidyanathan et al. [16] and this is continued to large and small porous effect by Sekar et al. [17, 18]. Reena and Rana [19] have been analyzed the thermosolutal convective instability of micropolar rotating fluids in a porous effect. They used the Darcy model. Chand [20] studied the porous effect on triple-diffusive convective instability in micropolar ferromagnetic fluid.

In present investigation, our intension is to consider salinity gradient on magnetization and magnetic potential equation and thermal convection problem in micropolar fluid of Eringen extend to the thermohaline convection in micropolar ferrofluid. Also, an effect of salinity gradient and how micropolar parameters affect the stability in micropolar ferromagnetic fluid heated from below and salted from above. The stationary and oscillatory instabilities are studied.
\end{abstract}

2.Mathematical Formulation Of Problem 


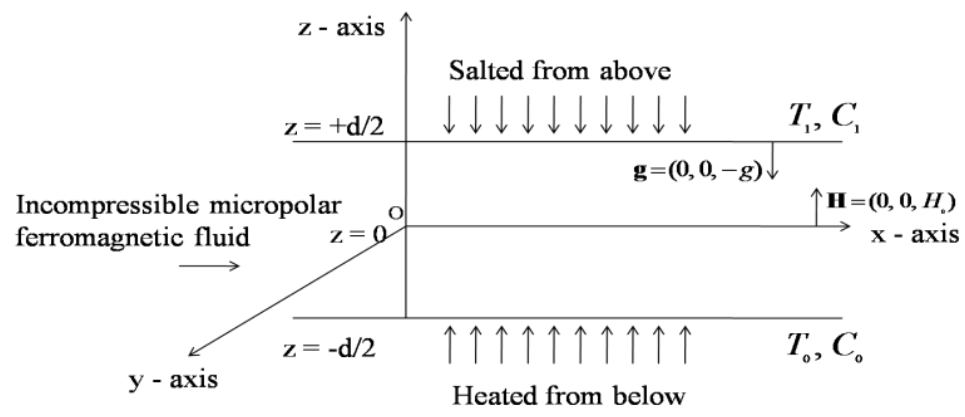

Fig.1 Geometrical configuration

Here we consider, an infinite horizontal micropolar ferrofluid layer heated from below and salted from above. The fluid layer is of thickness $d$ and the fluid is considered as an electrically non-conducting incompressible one. The temperature and salinity at the bottom and top surfaces $z=\mp d / 2$ are $T_{0} \pm(D T) / 2$ and $S_{0} \mp(D S) / 2$, respectively and $\beta_{t}(=|d T / d z|)$ and $\beta_{s}(=|d S / d z|)$ are maintained. The governing equations are

$\nabla \cdot \mathbf{q}=0$

The continuity equation is

The momentum and internal angular momentum equations are

$\rho_{0}\left(\frac{\partial}{\partial t}+(\mathbf{q} \cdot \nabla)\right) \mathbf{q}=-\nabla p+\rho \mathbf{g}+\nabla .(\mathbf{H B})+2 \zeta(\nabla \times \boldsymbol{\omega})+(\zeta+\eta) \nabla^{2} \mathbf{q}$
$\rho_{0} I\left(\frac{\partial}{\partial t}+(\mathbf{q} \cdot \nabla)\right) \boldsymbol{\omega}=2 \zeta(\nabla \times \mathbf{q}-2 \boldsymbol{\omega})+\mu_{0}(\mathbf{M} \times \mathbf{H})+\left(\lambda^{\prime}+\eta^{\prime}\right) \nabla(\nabla \cdot \boldsymbol{\omega})+\eta^{\prime} \nabla^{2} \boldsymbol{\omega}$

The temperature equation is

$\left[\rho_{0} C_{v, H}-\mu_{o} \mathbf{H} \cdot\left(\frac{\partial \mathbf{M}}{\partial T}\right)_{v, H}\right] \frac{D T}{D t}+\rho_{s} C_{s}\left(\frac{\partial T}{\partial t}\right)+\mu_{0} T\left(\frac{\partial \mathbf{M}}{\partial T}\right)_{v, H} . \frac{D \mathbf{H}}{D t}=K_{1} \nabla^{2} T+\delta(\nabla \times \boldsymbol{\omega}) . \nabla T+\phi$

The mass flux equation is

$\rho_{0}(\partial / \partial t+\mathbf{q} . \nabla) S=K_{S} \nabla^{2} S$

We can assume the magnetization using Maxwell's equation for non-conducting fluids [16-18] is $\mathbf{M}=\mathbf{H} M(H, T, S) / H \cdot$ The linearized magnetic equation in term of $H_{0}, T_{a}$ and $S_{a}$ is

$M=M_{0}+\chi\left(\mathrm{H}-H_{0}\right)-K\left(T-T_{a}\right)+K_{2}\left(S-S_{a}\right)$

The density equation of state is

$\rho=\rho_{0}\left[1-\alpha_{t}\left(T-T_{a}\right)+\alpha_{S}\left(S-S_{a}\right)\right]$

where $\mathbf{q}$ - velocity of fluid, $\rho_{0}$ - mean density of the clean fluid, $p$ - pressure, $\rho$ - density of the fluid, $\mathbf{g}$ gravitational field, H-magnetic field, B-magnetic induction, $\zeta$-coupling viscosity, $\omega_{\text {-microrotation, }} \eta_{\text {-shear }}$ viscosity coefficient, $I$-moment of inertia, M-magnetization, $\lambda^{\prime}$-bulk spin viscosity, $\eta^{\prime}$-shear spin viscosity, $C_{v, H}$-effective heat capacity at constant volume, $C_{s}$-specific heat solid material, ${ }^{\mu_{0}}$-viscosity of the fluid when the applied magnetic field is absent, $K_{1}$-thermal diffusivity, $T$-temperature, $\delta$-micropolar heat conduction coefficient, $S$-solute concentration, $K_{s}$-concentration diffusivity, $H_{0}$-uniform magnetic field, $T_{a}$ average temperature, $S_{a}$-average salinity, $\alpha_{t}$-thermal expansion coefficient and $\alpha_{s}$-analogous solvent coefficient.

The basic state quantities are 


$$
\begin{aligned}
& \mathbf{M}_{b}(z)=\left[M_{0}+\frac{K \beta_{t} z}{1+\chi}-\frac{K_{2} \beta_{S} z}{1+\chi}\right] \mathbf{k}, \rho(z)=\rho_{0}\left[1+\alpha_{t} \beta_{t} z-\alpha_{S} \beta_{S} z\right], \mathbf{q}=\mathbf{q}_{b}=\left(\begin{array}{lll}
0, & 0, & 0
\end{array}\right), \\
& \mathbf{H}_{b}(z)=\left[H_{0}-\frac{K \beta_{t} z}{1+\chi}+\frac{K_{2} \beta_{S} z}{1+\chi}\right] \mathbf{k}, \quad \boldsymbol{\omega}=\boldsymbol{\omega}_{b}=\left(\begin{array}{lll}
0, & 0,0
\end{array}\right), \\
& T_{b}=T_{0}-\beta_{t} z, S_{b}=S_{0}-\beta_{S} z, p=p_{b}(z) \text { and } M_{0}+H_{0}=H_{0}^{e x t} \text {. }
\end{aligned}
$$

A small thermal disturbance is made on the system. Let us take the perturbed components of $\mathbf{M}$ and $\mathbf{H}$ be $\left[M_{1}^{\prime}, M_{2}^{\prime}, M_{0}(z)+M_{3}^{\prime}\right]$ and $\left[H_{1}^{\prime}, H_{2}^{\prime}, H_{0}(z)+H_{3}^{\prime}\right]$, respectively. The perturbed quantities are

$\mathbf{H}=\mathbf{H}_{b}(z)+\mathbf{H}^{\prime}, \boldsymbol{\omega}=\boldsymbol{\omega}_{b}+\boldsymbol{\omega}^{\prime}, \quad p=p_{b}(z)+p^{\prime}, \quad S=S_{b}(\mathrm{z})+S^{\prime}$,

$\mathbf{M}=\mathbf{M}_{b}(z)+\mathbf{M}^{\prime}, \mathbf{q}=\mathbf{q}_{b}+\mathbf{q}^{\prime}, \rho=\rho_{b}+\rho^{\prime}, T=T_{b}(\mathrm{z})+\theta$,

the superscript 'denotes perturbed state.

The perturbed density equation can be calculated as $\rho^{\prime}=\rho_{0}\left(-\alpha_{t} \theta+\alpha_{s} S^{\prime}\right)$

\section{1. normal mode analysis method}

We undertake the perturbation quantities by use of normal modes are $w(x, y, z, t)=w(z, t) \exp \left[i k_{x} x+i k_{y} y\right]$

$$
\begin{aligned}
& \theta(x, y, z, t)=\theta(z, t) \exp \left[i k_{x} x+i k_{y} y\right] \\
& \phi(x, y, z, t)=\phi(z, t) \exp \left[i k_{x} x+i k_{y} y\right] \\
& S(x, y, z, t)=S(z, t) \exp \left[i k_{x} x+i k_{y} y\right]
\end{aligned}
$$

In Eq. (2), one can get the $\mathrm{k}^{\text {th }}$ component is

$$
\left.\begin{array}{rl}
\left(\rho_{0} \frac{\partial}{\partial t}\left(\frac{\partial^{2}}{\partial z^{2}}-k_{0}^{2}\right)\right) w & \left.=\mu_{0} K \beta_{t} k_{0}^{2} \frac{\partial \phi}{\partial z}-\left(\frac{\mu_{0} K^{2} \beta_{t}}{1+\chi}\right) k_{0}^{2} \theta+\left(\frac{\mu_{0} K K_{2} \beta_{t}}{1+\chi}\right) k_{0}^{2} S^{\prime}-\mu_{0} K_{2} \beta_{S} k_{0}^{2} \frac{\partial \phi}{\partial z}+\left(\frac{\mu_{0} K K_{2} \beta_{S}}{1+\chi}\right) k_{0}^{2} \theta\right) \\
& -\left(\frac{\mu_{0} K_{2}^{2} \beta_{S}}{1+\chi}\right) k_{0}^{2} S^{\prime}-\rho_{0} g \alpha_{t} k_{0}^{2} \theta+\rho_{0} g \alpha_{S} k_{0}^{2} S+2 \zeta\left(\frac{\partial^{2}}{\partial z^{2}}-k_{0}^{2}\right) \Omega_{3}^{\prime}+(\zeta+\eta)\left(\frac{\partial^{2}}{\partial z^{2}}-k_{0}^{2}\right)^{2} w
\end{array}\right\}
$$

Internal angular Eq. (3) can be manipulated as

$$
\rho_{0} I \frac{\partial \Omega_{3}^{\prime}}{\partial t}=-2 \zeta\left[\left(\frac{\partial^{2}}{\partial z^{2}}-k_{0}^{2}\right) w+2 \Omega_{3}^{\prime}\right]+\eta^{\prime}\left(\frac{\partial^{2}}{\partial z^{2}}-k_{0}^{2}\right) \Omega_{3}^{\prime}
$$

Eq. (4) can be calculated as

$\rho C_{1} \frac{\partial \theta}{\partial t}-\mu_{0} K T_{0} \frac{\partial}{\partial t}\left(\frac{\partial \phi}{\partial z}\right)=K_{1}\left(\frac{\partial^{2}}{\partial z}-k_{0}^{2}\right) \theta+\left[\rho C_{2} \beta_{t}-\left(\frac{\mu_{0} K^{2} T_{0}^{2} \beta_{t}}{1+\chi}\right)+\left(\frac{\mu_{0} K K_{2} T_{0} \beta_{S}}{1+\chi}\right)\right] w-\delta \beta_{t} \Omega_{3}^{\prime}$

The Salinity equation is

$(\partial / \partial t) S+\beta_{S} w=K_{S}\left(\left(\partial^{2} / \partial z^{2}\right)-k_{0}^{2}\right) S^{\prime}$

Using Vaidyanathan et al. [14], one gets

$$
(1+\chi) \frac{\partial^{2} \phi}{\partial z^{2}}-\left(1+\frac{M_{0}}{H_{0}}\right) k_{0}^{2} \phi-K \frac{\partial \theta}{\partial z}+K_{2} \frac{\partial S}{\partial z}=0
$$

The non-dimensional equations can be derived by use of normal mode method as 


$$
\begin{aligned}
& \left.\left(\frac{\partial}{\partial t^{*}}\left(D^{2}-a^{2}\right)\right) w^{*}=a M_{1} R^{1 / 2} D \phi^{*}-a\left(1+M_{1}\right) R^{1 / 2} T^{*}+a R^{1 / 2} M_{1} M_{5} D \phi^{*}\right) \\
& +a R_{S}^{1 / 2}\left(1+M_{4}\right) S^{*}-a R^{1 / 2} M_{1} M_{5} T^{*}+a R_{S}^{1 / 2} \frac{M_{4}}{M_{5}} S^{*} \\
& +2 N_{1}\left(D^{2}-a^{2}\right) \Omega_{3}^{* *}+(\zeta+\eta)\left(D^{2}-a^{2}\right)^{2} w^{*} \\
& I^{\prime} \frac{\partial \Omega_{3}^{*}}{\partial t}=-2\left[\left(D^{2}-a^{2}\right) w^{*}+2 \Omega_{3}^{*}\right] N_{1}+\left(D^{2}-a^{2}\right) N_{3}^{\prime} \Omega_{3}^{\prime *} \\
& {\left[P_{r}^{\prime} \frac{\partial T^{*}}{\partial t^{*}}-M_{2} P_{r} \frac{\partial}{\partial t^{*}}\left(D \phi^{*}\right)\right]=\left(D^{2}-a^{2}\right) T^{*}+a\left(1-M_{2}-M_{2} M_{5}\right) R^{1 / 2} w^{*}-a N_{5}^{\prime} R^{1 / 2} \Omega_{3}^{* *}} \\
& P_{r} \frac{\partial S^{*}}{\partial t^{*}}=\tau\left(D^{2}-a^{2}\right) S^{*}-a R_{S}^{1 / 2}\left(\frac{M_{5}}{M_{6}}\right) w^{*}, \\
& D^{2} \phi^{*}-M_{3} a^{2} \phi^{*}-D T^{*}+\frac{M_{5}}{\tau}\left(\frac{R}{R_{S}}\right)^{1 / 2} D S^{*}=0,
\end{aligned}
$$

where the dimensionless quantities are

$$
\left.\begin{array}{l}
w^{*}=\frac{w d}{v}, t^{*}=\frac{v t}{d^{2}}, T^{*}=\left(\frac{K_{1} a R^{1 / 2}}{\rho_{0} C_{v, H} \beta_{t} v d}\right) \theta, \phi^{*}=\left(\frac{(1+\chi) K_{1} a R^{1 / 2}}{\rho_{0} C_{v, H} K \beta_{t} v d^{2}}\right) \phi, \quad z^{*}=\frac{z}{d}, a=k_{0} d, \quad D=\frac{\partial}{\partial z^{*}}, \\
S^{*}=\left(\frac{K_{S} a R_{S}^{1 / 2}}{\rho_{0} C_{v, H} \beta_{S} v d}\right) S, \quad v=\frac{\mu}{\rho_{0}}, \Omega_{3}^{*}=\frac{\Omega_{3}}{v} d^{3} M_{1}=\frac{\mu_{0} K^{2} \beta_{t}}{(1+\chi) \rho_{0} g \alpha_{t}}, M_{2}=\frac{\mu_{0} K^{2} T}{(1+\chi) \rho_{0} C_{v, H}}, N_{1}=\frac{\zeta}{\eta}, \\
M_{3}=\frac{1+M_{0} / H_{0}}{(1+\chi)}, M_{4}=\frac{\mu_{0} K^{2} \beta_{s}}{(1+\chi) \rho_{0} g \alpha_{s}}, M_{5}=\frac{K_{2} \beta_{s}}{K \beta_{t}}, M_{6}=\frac{K_{S}}{K_{1}}, \tau=\rho_{0} C_{v, H}\left(\frac{K_{S}}{K_{1}}\right), N_{3}^{\prime}=\frac{\eta^{\prime}}{\eta d^{2}}, \\
N_{5}^{\prime}=\frac{\delta}{\rho C_{2} d^{2}}, I^{\prime}=\frac{I}{d^{2}}, P_{r}=\frac{v}{K_{1}} \rho C_{2}, P_{r}^{\prime}=\frac{v}{K_{1}} \rho C_{1}, R_{S}=\frac{\rho_{0} C_{v, H} \beta_{s} \alpha_{s} g d^{4}}{v K_{S}}, R=\frac{\rho_{0} C_{v, H} \beta_{t} \alpha_{t} g d^{4}}{v K_{1}}
\end{array}\right\}
$$

\section{2. linear stability analysis}

The stationary and oscillatory instabilities have been studied using linear theory. The boundary conditions are $w^{*}=D^{2} w^{*}=D \phi^{*}=S^{*}=\Omega_{3}^{*}=T^{*}=0$ at $z^{*}= \pm 1 / 2$.

The exact solutions satisfying above Eq. (23) are

$$
\left.\begin{array}{l}
w^{*}=X_{1} e^{\sigma t^{*}} \cos \pi z^{*}, \quad T^{*}=X_{2} e^{\sigma t^{*}} \cos \pi z^{*}, \quad S^{*}=X_{3} e^{\sigma t^{*}} \cos \pi z^{*} \\
D \phi^{*}=X_{4} e^{\sigma t^{*}} \cos \pi z^{*}, \quad \phi^{*}=\frac{X_{4}}{\pi} \sin \pi z^{*}, \Omega_{3}^{*}=X_{5} e^{\sigma t^{*}} \cos \pi z^{*}
\end{array}\right\}
$$

where $X_{1}, X_{2}, X_{3}, X_{4}$ and $X_{5}$ are constants. Eqs. (17)-(21) can be mathematical manipulated using Eq. (23) as

$$
\begin{aligned}
& \left.\begin{array}{rl}
\left(\pi^{2}+a^{2}\right)[ & \left.\sigma+\left(1+N_{1}\right)\left(\pi^{2}+a^{2}\right)^{2}\right] X_{1}-a R^{1 / 2}\left[1+\left(1+M_{5}\right) M_{1}\right] X_{2} \\
& +a\left(1+M_{4}+M_{4} M_{5}^{-1}\right) R_{S}^{1 / 2} X_{3}+a\left(1+M_{5}\right) R^{1 / 2} M_{1} X_{4}-2\left(\pi^{2}+a^{2}\right) N_{1} X_{5}=0,
\end{array}\right\} \\
& -2\left(\pi^{2}+a^{2}\right) N_{1} X_{1}+\left[4 N_{1}+\left(\pi^{2}+a^{2}\right) N_{3}^{\prime}+I^{\prime} \sigma\right] X_{5}=0, \\
& a\left(1-M_{2}-M_{2} M_{5}\right) R^{1 / 2} X_{1}-\left(P_{r} \sigma+\pi^{2}+a^{2}\right) X_{2}+P_{r} \varepsilon \sigma M_{2} X_{4}-a N_{5}^{\prime} R^{1 / 2} X_{5}=0, \\
& a M_{6} R_{S}^{1 / 2} X_{1}+\left[\left(\pi^{2}+a^{2}\right) \tau+\sigma P_{r}\right] X_{3}=0, \\
& -R_{S}^{1 / 2} \pi^{2} X_{2}+R^{1 / 2} \pi^{2}\left(M_{5} / M_{6}^{-1}\right) X_{3}+R_{S}^{1 / 2}\left(\pi^{2}+a^{2} M_{3}\right) X_{5}=0,
\end{aligned}
$$


To evaluate the Eigen function, determination of the co-efficient of $X_{1}, X_{2}, X_{3}, X_{4}$ and $X_{5}$ in Eqs. (25)-(29) is equal to zero. Using the analyses Vaidyanathan et al. [13, 14], Eqs. (25)-(29) have been adopted to get

$T_{1} \sigma^{4}+T_{2} \sigma^{3}+T_{3} \sigma^{2}+T_{4} \sigma+T_{5}=0$

where

$$
\begin{aligned}
T_{1}= & P_{r}^{\prime}\left(I^{\prime}-x_{1}\right), T_{2}=P_{r}^{\prime} I^{\prime} x_{1}^{2}\left(1+P_{r}^{\prime}\right)-P_{r}^{\prime} x_{1}\left(P_{r}^{\prime}+I^{\prime}\right) \\
T_{3}= & a^{2} x_{4} P_{r}^{\prime} I^{\prime} R-x_{8} x_{6}^{2} P_{r}^{\prime^{2}}+x_{1}^{2}\left(1+P_{r}^{\prime}\right)\left(P_{r}^{\prime}+I^{\prime}\right)+x_{8} P_{r}^{\prime} I^{\prime}\left(a^{2} x_{2}-x_{1}^{2}\right)-x_{1}^{2} x_{6} x_{7} \tau P_{r}^{\prime} \\
& +a^{2} M_{6} P_{r}^{\prime} I^{\prime} R\left(x_{4} x_{9}+x_{3} x_{8}\right) \\
T_{4}= & -a^{2} \pi^{2} \tau x_{1} x_{4} I^{\prime} R-a^{2} \pi^{2} P_{r}^{\prime} x_{4} R\left(x_{7}-x_{6} N_{5}^{\prime}\right)-x_{8} x_{6} P_{r}^{\prime}\left(a^{2} x_{2} N_{5}^{\prime} R+x_{1} x_{6}\right)-x_{1} x_{6}^{2} x_{8} \tau P_{r}^{\prime} \\
& +\left(P_{r}^{\prime}+I^{\prime}\right)\left(a^{2} x_{2} R-x_{1}^{2}\right)-x_{1}^{3} x_{7} x_{8} \tau\left(1+P_{r}^{\prime}\right)+\left(P_{r}^{\prime} x_{7}-x_{1} I^{\prime}\right)\left(a^{2} M_{6} x_{3} x_{8}-a^{2} M_{6} x_{4} x_{9} R\right) \\
T_{5}= & x_{1} \tau\left(\pi^{2}\left(a^{2} x_{4} x_{7} R-a^{2} x_{4} x_{6} N_{5}^{\prime} R\right)+x_{8}\left(x_{6}\left(a^{2} x_{2} R+x_{1} x_{6}\right)-x_{7}\left(a^{2} x_{2} R-x_{1}^{3}\right)\right)\right) \\
& -a^{2} x_{7} M_{6}\left(x_{1} x_{4} x_{9} R+x_{1} x_{3} x_{9} R_{s}\right) \\
x_{1}= & \pi^{2}+a^{2}, x_{2}=1+x_{4}, x_{3}=1+M_{4}+\left(M_{4} / M_{5}\right), x_{4}=M_{1}\left(1+M_{5}\right), \\
x_{5}= & 2 N_{1}, x_{6}=x_{1} x_{5}, x_{7}=4 N_{1}+x_{1} N_{3}^{\prime}, x_{8}=\pi^{2}+a^{2} M_{3}, x_{9}=M_{5} \pi^{2} / M_{6}
\end{aligned}
$$

\subsection{The case of stationary instability}

For steady state, we have $\sigma=0$ at the marginal stability. Then the Eq. (30) leads to get Eigen value $R_{s c}$ for which solution exists. Using the analyses [14]-[15], the critical magnetic Rayleigh number $R_{s c}$ has been obtained using

$$
R_{s c}=\frac{N r}{D r}
$$

where

$$
\begin{aligned}
N r= & \left(\pi^{2}+a^{2}\right)^{3}\left(\left(4 N_{1}+\left(\pi^{2}+a^{2}\right) N_{3}^{\prime}\right)\left(1+N_{1}\right)-4 N_{1}^{2}\right)-a^{2}\left(1+M_{4}+M_{4} M_{5}^{-1}\right)\left(4 N_{1}+\left(\pi^{2}+a^{2}\right) N_{3}^{\prime}\right) M_{6} R_{s} \tau^{-1} \\
D r= & a^{2}\left(1+M_{1}\left(1+M_{5}\right)\right)\left(4 N_{1}+\left(\pi^{2}+a^{2}\right)\left(N_{3}^{\prime}-2 N_{1} N_{5}^{\prime}\right)\right) \\
& -\frac{a^{2} \pi^{2} M_{1}\left(1+M_{5}\right)}{\left(\pi^{2}+a^{2} M_{3}\right)}\left(\left(4 N_{1}+\left(\pi^{2}+a^{2}\right) N_{3}^{\prime}\right)\left(1+M_{5} \tau^{-1}\right)-2 N_{1} N_{5}^{\prime}\left(\pi^{2}+a^{2}\right)\right)
\end{aligned}
$$

When $M_{1}$ is very large, one can gets $N_{\mathrm{sc}}\left(=M_{1} R_{\mathrm{sc}}\right)$.

$N_{s c}=\frac{N r}{D r}$

where

$$
\begin{aligned}
\operatorname{Dr}= & a^{2}\left(1+M_{5}\right)\left(4 N_{1}+\left(\pi^{2}+a^{2}\right)\left(N_{3}^{\prime}-2 N_{1} N_{5}^{\prime}\right)\right) \\
& -\frac{a^{2}\left(1+M_{5}\right) \pi^{2}}{\left(\pi^{2}+a^{2} M_{3}\right)}\left(\left(4 N_{1}+\left(\pi^{2}+a^{2}\right) N_{3}^{\prime}\right)\left(1+M_{5} \tau^{-1}\right)-2 N_{1} N_{5}^{\prime}\left(\pi^{2}+a^{2}\right)\right)
\end{aligned}
$$

Here $a$ is denoted as critical wave number $a_{\mathrm{c}}$. Analysis of the classical results is given below:

Assuming $\varepsilon=1, N_{3}^{\prime}=1, N_{1}=0$, and $N_{5}^{\prime}=0$ in Eq. (32), one get

$$
N_{s c}=\frac{\left(\pi^{2}+a^{2}\right)^{3}-a^{2}\left(1+M_{4}+M_{4} M_{5}^{-1}\right) M_{6} \tau^{-1} R_{s}}{a^{2}\left(1+M_{5}\right)\left(1-\pi^{2}\left(1+M_{5} \tau^{-1}\right) /\left(\pi^{2}+a^{2} M_{3}\right)\right)}
$$

which is an expression for $N_{\mathrm{sc}}$ of Vaidyanathan et al. [13].

Moreover, if $M_{4}, M_{6}, \tau^{-1}, R_{S}=0$ in Eq. (33), it gives $N_{\mathrm{sc}}$ of the Finlayson [3], a single component fluid.

\subsection{The case of oscillatory instability}




$$
\begin{array}{r}
\text { Taking } \sigma=i \sigma_{1} \quad\left(\sigma_{1}>0\right) \text { in Eq. (30), it leads to } R_{o c} \text { has been derived using } \\
R_{o c}=\left(T_{1} Y_{2} \sigma_{1}^{6}+\left(T_{1} Y_{5}+T_{2} Y_{3}\right) \sigma_{1}^{4}+\left(Y_{2}\left(Y_{2}+Y_{6}\right)+Y_{3} Y_{4}\right) \sigma_{1}^{2}+Y_{5}\left(Y_{2}+Y_{6}\right)\right) / D r
\end{array}
$$

where

$$
\begin{aligned}
Y_{1}= & a^{2} M_{6} P_{r}^{\prime} I^{\prime}\left(x_{4} x_{9}+x_{3} x_{8}\right)-a^{2} x_{4} P_{r}^{\prime} I^{\prime}-a^{2} x_{2} x_{8} P_{r}^{\prime} I^{\prime}, \\
Y_{2}= & x_{1}^{2}\left(1+P_{r}^{\prime}\right)\left(P_{r}^{\prime}+I^{\prime}\right)-x_{8} x_{6}^{2} P_{r}^{2}-x_{1}^{3} x_{8} P_{r}^{\prime} I^{\prime}-P_{r}^{\prime} \tau x_{1}^{2} x_{8} x_{7}, \\
Y_{3}= & -a^{2} \pi^{2} I^{\prime} \tau x_{1} x_{4}-a^{2} \pi^{2} P_{r}^{\prime} x_{4}\left(x_{7}-x_{6} N_{5}^{\prime}\right)-a^{2} x_{3} x_{6} P_{r}^{\prime} x_{2} N_{5}^{\prime}-x_{1} x_{6}^{2} x_{8} \tau P_{r}^{\prime}+a^{2} x_{2}\left(P_{r}^{\prime}+I^{\prime}\right) \\
& -a^{2} M_{6} x_{4} x_{9}\left(P_{r}^{\prime} x_{7}-x_{1} I^{\prime}\right), \\
Y_{4}= & -x_{8} x_{6} P_{r}^{\prime} \tau-\left(P_{r}^{\prime}+I^{\prime}\right) x_{1}^{2}+x_{1}^{3} x_{7} x_{8} \tau\left(1+P_{r}^{\prime}\right)-\left(P_{r}^{\prime} x_{7}-x_{1} I^{\prime}\right) a^{2} M_{6} x_{3} x_{8} R_{s}, \\
Y_{5}= & x_{1} \tau \pi^{2}\left(a^{2} x_{4} x_{7}-a^{2} x_{4} x_{6} N_{5}^{\prime}\right)+a^{2} x_{2} x_{6} x_{8} N_{5}^{\prime}+a^{2} x_{2} x_{6} x_{7} x_{8}+a^{2} M_{6} x_{1} x_{4} x_{7} x_{9}, \\
Y_{6}= & \tau x_{1}^{4} x_{7} x_{8}+a^{2} M_{6} P_{r}^{\prime} x_{7} x_{8}-\tau x_{1}^{2} x_{6}^{2} x_{8}, \\
D r= & \left(Y_{2} \sigma_{1}^{2}+Y_{5}\right)^{2}-\sigma_{1}^{2} Y_{3}^{2}, \sigma_{1}^{2}=\left(-A_{2} \pm \sqrt{A_{2}^{2}-4 A_{1} A_{3}}\right) / 2 A_{1}, \\
A_{1}= & T_{2} Y_{2}+T_{1} Y_{3}, A_{2}=T_{2} Y_{5}+Y_{4} Y_{2}, \text { and } A_{3}=Y_{4} Y_{5}-Y_{3}\left(Y_{2}+Y_{6}\right)
\end{aligned}
$$

\section{Discussion of Results}

In this investigation, thermohaline convection in micropolar ferrofluid layer is studied. The fluid layer is heated from below and salted from above and the convective system is subjected to a transverse uniform magnetic field. The thermal perturbations and linear stability analysis are used in the study. Here we consider the free boundary conditions. The magnetic numbers $M_{1}$ and $M_{2}$ are considered the values 1000 and 0 , respectively. $M_{3}$ is taken from 5 to 25 (Vaidyanthan et al. [14]) and $\tau$ ranges from 0.05 to 0.11 (Vaidyanthan et al. [14]) and $R_{\mathrm{S}}$ taken from -500 to 500. The magnetization parameters $M_{4}$ and $M_{6}$ are taken to be 0.1 and $M_{5}=$ 0.5 (Vaidyanthan et al. [16]). Further, $N_{1}, N_{3}^{\prime}$ and $N_{5}^{\prime}$ are taken to be non-negative values which is presented by Eringen [21] and he assumed the clausius-Duhem inequality. $P_{\mathrm{r}}$ is taken as 0.01 .

The variation of $N_{\mathrm{sc}}$ with the coupling parameter $N_{1}$ is depicted in Fig. 2 (a) and (b). It is observed from the Fig. 2 (a) that the convective system gives stabilizing behavior, when increasing values of $M_{3}$ and $R_{\mathrm{S}}$. Due to the increasing value of $N_{1}$ from 0 to 1 and $R_{\mathrm{S}}$ from -500 to 500 on the system, $N_{\mathrm{sc}}$ gets the highest values and the system has more stabilizing effect. But, an increasing of $M_{3}$ from 5 to 25 , the system shows the stabilizing effect and it is less pronounced. $M_{3}$ analyzed for destabilizing behavior always [13-14, 16-17]. But, introducing of $N_{1}$ on $M_{3}$, the system gets stabilizing effect. Fig. 2 (b) represents the plot of $N_{\mathrm{sc}}$ versus $N_{1}$ for different $\tau$. This figure shows that $N_{1}$ has the stabilizing behavior for increasing value of $\tau$. This is because, the greater the mass and heat transports and more buoyancy energy, which contributes to thermal instability. Also, it is shown from the Fig. 2 (c) that the increase in $N_{1}$ stabilizes the system for increasing of $\tau$ and $R_{\mathrm{S}}$. Also, in the presence of $R_{\mathrm{S}}$ $=500, a_{\mathrm{c}}$ is close to zero. In this moment, the system has an equilibrium state.

Figs. 3 (a) and (b) display the variation of $N_{\mathrm{sc}}$ versus spin diffusion parameter ${ }^{\prime}{ }^{\prime}$ for increasing of $R_{\mathrm{S}}$ and $M_{3}$ and $\tau$, respectively. In Fig. 3 (a), we observe that $N_{\text {sc }}$ decreases with increasing of $N_{3}$, which leads to destabilize the system. Moreover, when $R_{\mathrm{S}}=500, N_{\mathrm{sc}}$ gets zero value. Therefore, the system has a null effect. From Fig. 3 (b), it is seen that as $N_{3}{ }^{\prime}$ increases from 2 to 8 , there is a decrease in $N_{\text {sc }}$ indicating destabilization for different $\tau$. Fig. 3 (c) shows the variation of $a_{\mathrm{c}}$ versus $N_{3}{ }^{\prime}$ for various $\tau, R_{\mathrm{S}}$ and $M_{3}$. When $\tau$ increases from 0.05 to $0.1, R_{\mathrm{S}}$ increases from -500 to 500 and $M_{3}$ increases from 5 to 25 , there is a decrease in $a_{\mathrm{c}}$. It is clear that there is a destabilization on the system which is not much pronounced and when $R_{\mathrm{S}}=500$, there is an oscillation in $a_{\mathrm{c}}$.

Figs. 4 (a) and (b) show the variation of $N_{\text {sc }}$ versus micropolar heat conduction parameter $N_{5}{ }^{\prime}$ for different $M_{3}, \tau$ and. It is clear from the Fig. 4 (a) that $N_{5}{ }^{\prime}$ leads to an increase in $N_{\text {sc. Therefore, }} N_{5}{ }^{\prime}$ has a stabilizing effect. It is very clear from the Fig. 4 (b) that increase in $N_{5}{ }^{\prime}$, it is stabilizing behavior for various $R_{\mathrm{S}}$.

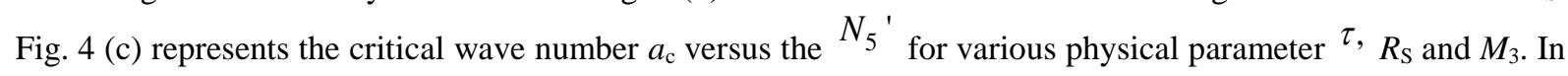
this figure $N_{5}{ }^{\prime}$ shows a stabilizing behavior. In such situation also the system ha no effect when $R_{\mathrm{S}}=500$. 
The increase in non-buoyancy magnetization parameter $M_{3}$ is obtained to cause large destabilization, because both thermal and magnetic mechanisms favor destabilization. This can be studied from Figs. 5 (a) and (b) in which the increase in $M_{3}$ and $\tau$, decrease in $N_{\mathrm{sc}}$ and $a_{\mathrm{c}}$, respectively.

From Fig. 6 (a), it is seen that an increase in $R_{\mathrm{S}}$, decrease in $N_{\mathrm{sc}}$. An increase of $R_{\mathrm{S}}$ would means that the system is salted from above. Also, when $R_{\mathrm{S}}=500$ and $\tau(=0.05,0.07,0.09)$, the $N_{\text {sc }}$ gets small values. But for the value $\tau=0.11$, suddenly $N_{\text {sc }}$ gets highest value. In this moment, the convective system gets stabilizing effect. Fig. 6 (b) shows the variation of $a_{\mathrm{c}}$ versus $R_{\mathrm{S}}$ for different $\tau$. When $R_{\mathrm{S}}$ increases from -500 to 500, there is a decrease in $a_{\text {c }}$ promoting instability. When the highest value of $R_{\mathrm{S}}(=500)$ the system tends to the same effect. That is, the system converges to the small values. But, when the highest values of $\tau(=0.11)$, the system has an equilibrium position.

\section{Conclusion}

In the present analysis, the results of a theoretical study on thermohaline convection in a micropolar ferrofluid are considered with free boundary conditions. We conclude that the effect of non-buoyancy magnetization $M_{3}$, salinity effect $R_{\mathrm{S}}$, spin diffusion parameter $N_{3}{ }^{\prime}$ have destabilizing behavior and the effect of coupling parameter $N_{1}$ and the micropolar heat conduction parameter $N_{5}{ }^{\prime}$ have a stabilizing effect due to the microrotation on the onset of convection.

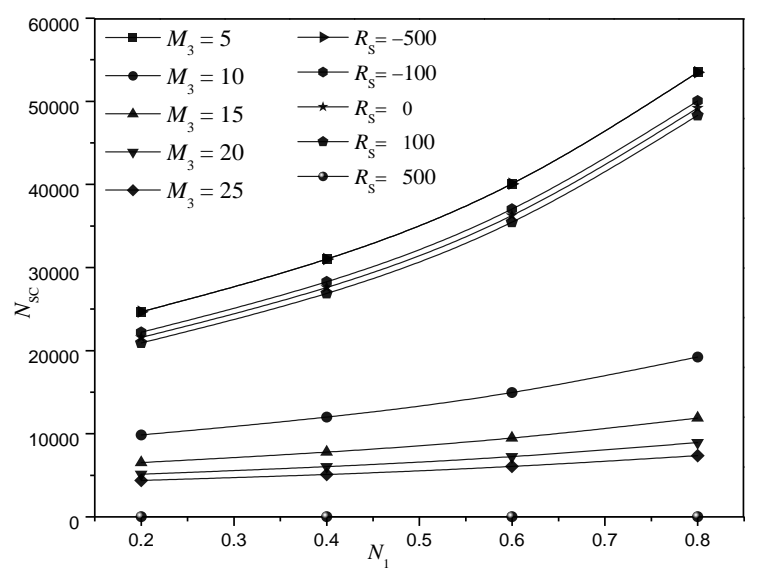

Fig. 2 (a). Variation of $N_{\mathrm{sc}}$ versus $N_{1}$ for different $M_{3}$ and $R_{\mathrm{S}}, N_{3}{ }^{\prime}=2, N_{5}{ }^{\prime}=0.2$ and $\tau=0.05$

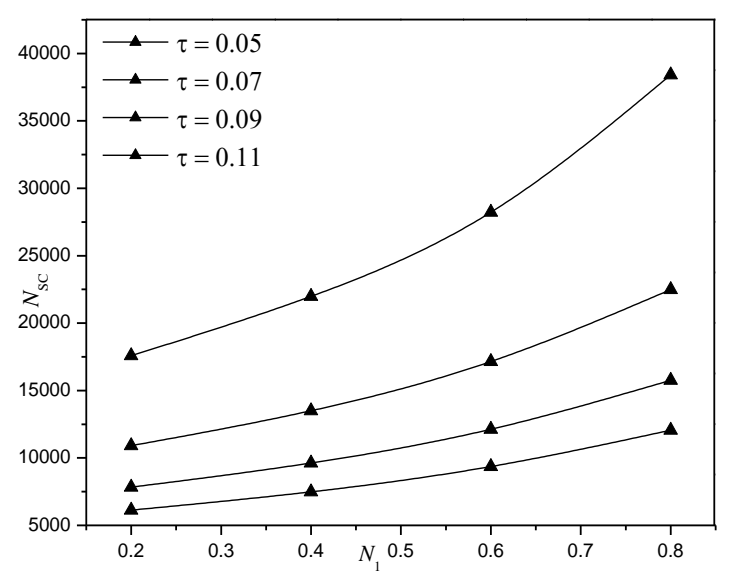

Fig. 2(b). Variation of $N_{\mathrm{sc}}$ versus $N_{1}$ for different $\tau, N_{3}{ }^{\prime}=2, N_{5}{ }^{\prime}=0.2, M_{3}=5$ and $R_{\mathrm{S}}=-500$.

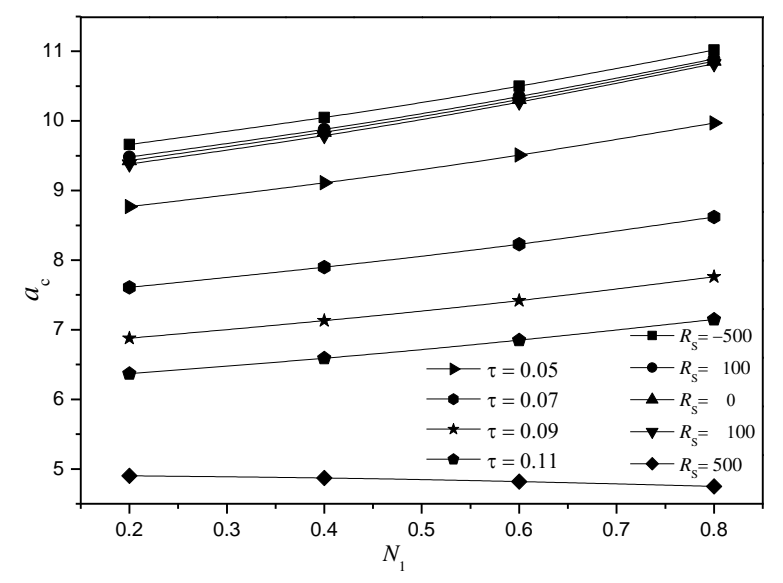

Fig. 2 (c). Variation of $a_{\mathrm{c}}$ versus $N_{1}$ for different $R_{\mathrm{S}}$ and $\tau, N_{3}{ }^{\prime}=2, N_{5}{ }^{\prime}=0.2$ and $M_{3}=5$. 


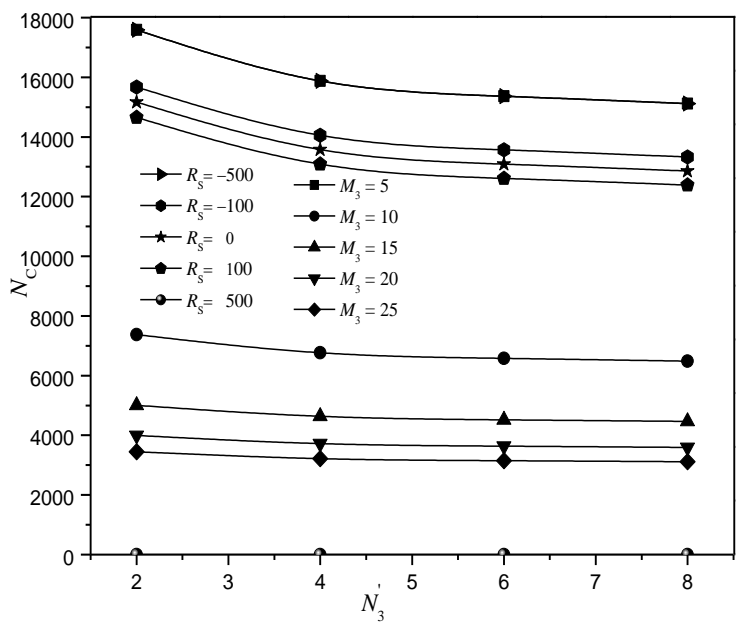

Fig. 3 (a). Variation of $N_{\mathrm{sc}}$ versus $N_{3}{ }^{\prime}$ for different $M_{3}$ and $R_{\mathrm{S}}, N_{1}=0.2, N_{5}{ }^{\prime}=0.2$ and $\tau=0.05$.

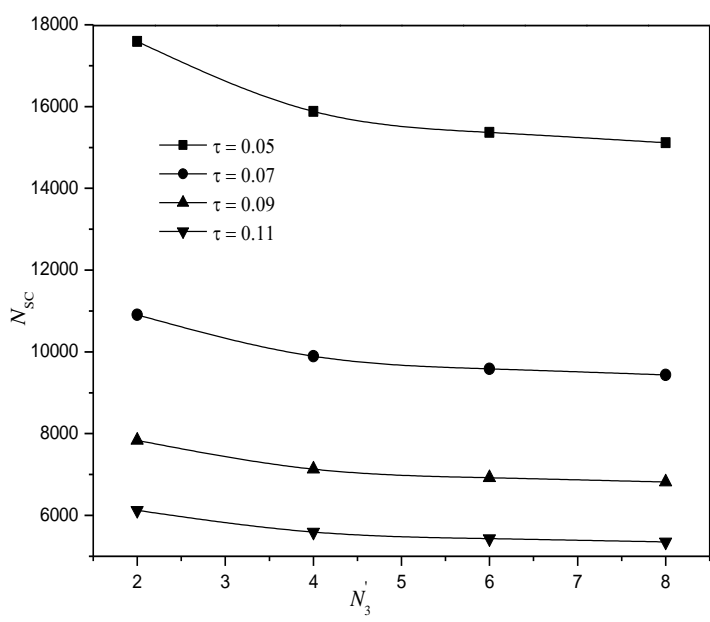

Fig. 3 (b). Variation of $N_{\mathrm{sc}}$ versus $N_{3}{ }^{\prime}$ for different $\tau, \quad N_{1}=0.2, N_{5}{ }^{\prime}=0.2, M_{3}=5$ and $R_{\mathrm{S}}=-500$.

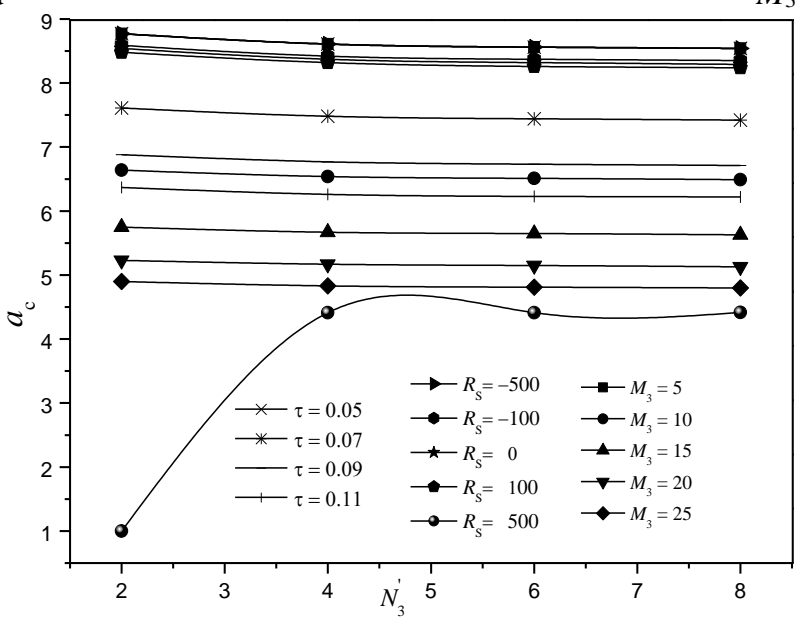

Fig. 3 (c) - Variation of $a_{\mathrm{c}}$ versus $N_{3}{ }^{\prime}$ for different $R_{\mathrm{S}}, M_{3}$ and $\tau, N_{1}=0.2, N_{5}{ }^{\prime}=0.2$ and $M_{3}=5$.

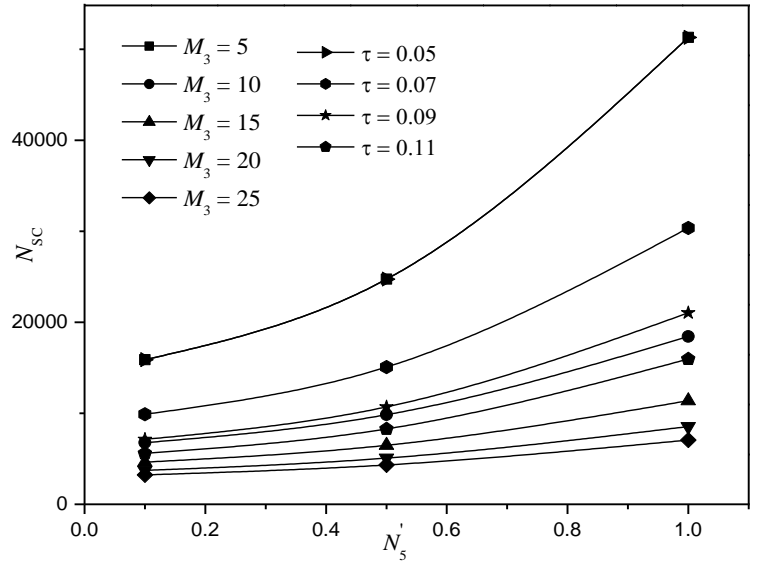

Fig. 4 (a). Variation of $N_{\mathrm{sc}}$ versus ${ }^{\prime}{ }^{\prime}$ for different $M_{3}$ and $\tau, N_{1}=0.2, N_{3}{ }^{\prime}=2$ and $R_{\mathrm{S}}=-500$.

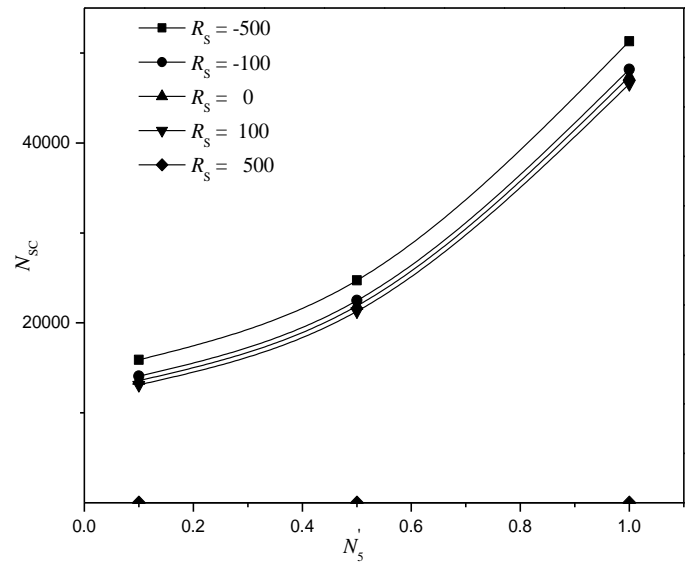

Fig. 4 (b). Variation of $N_{\mathrm{sc}}$ versus $N_{5}{ }^{\prime}$ for different $R_{\mathrm{S}}, \tau=0.05, N_{1}=0.2, N_{3}{ }^{\prime}=2, M_{3}=5$ and $R_{\mathrm{S}}=-500$. 


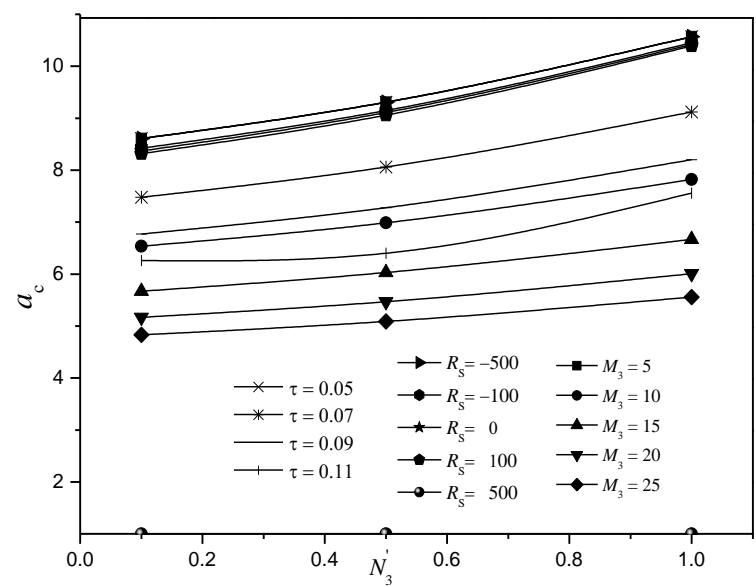

Fig. 4 (c). Variation of $a_{\mathrm{c}}$ versus $N_{5}{ }^{\prime}$ for different $R_{\mathrm{S}}, M_{3}$ and $\tau, N_{1}=0.2, N_{5}{ }^{\prime}=0.2$ and $M_{3}=5$.

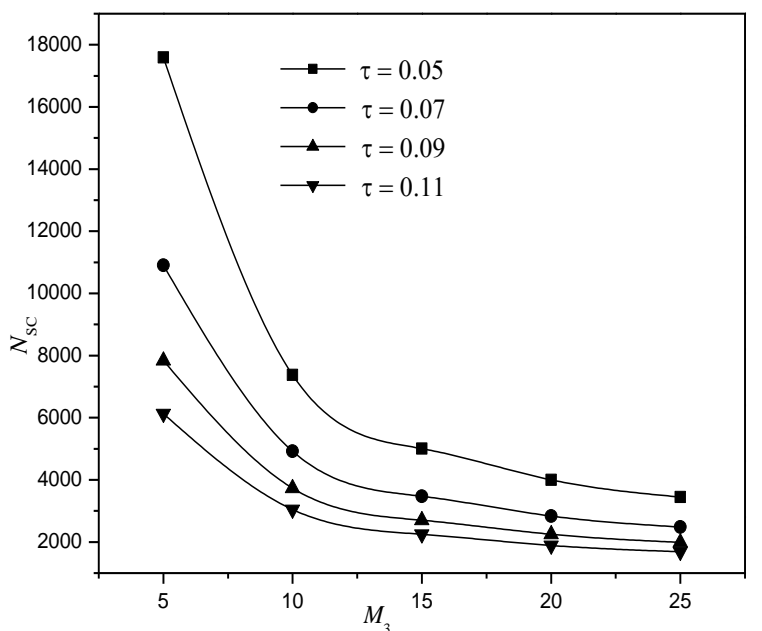

Fig. 5 (a). Variation of $N_{\mathrm{sc}}$ versus $M_{3}$ for different $\tau$, $N_{1}=0.2, N_{3}{ }^{\prime}=2, N_{5}{ }^{\prime}=0.2$ and $R_{\mathrm{S}}=-500$.

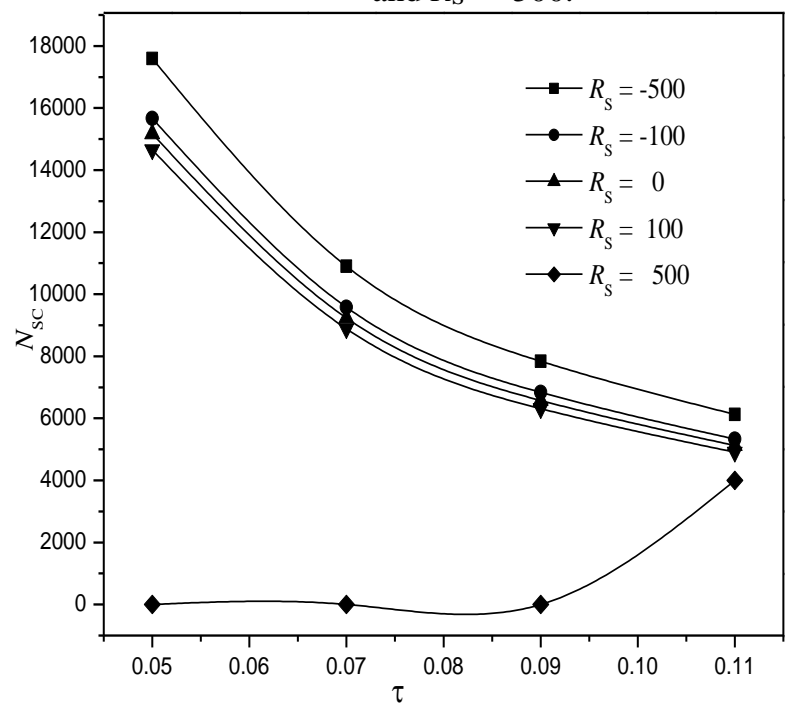

Fig. 6 (a). Variation of $N_{\mathrm{sc}}$ versus $\tau$ for different $R_{\mathrm{S}}$, $N_{1}=0.2, N_{3}{ }^{\prime}=2, N_{5}{ }^{\prime}=0.2$ and $M_{3}=5$.

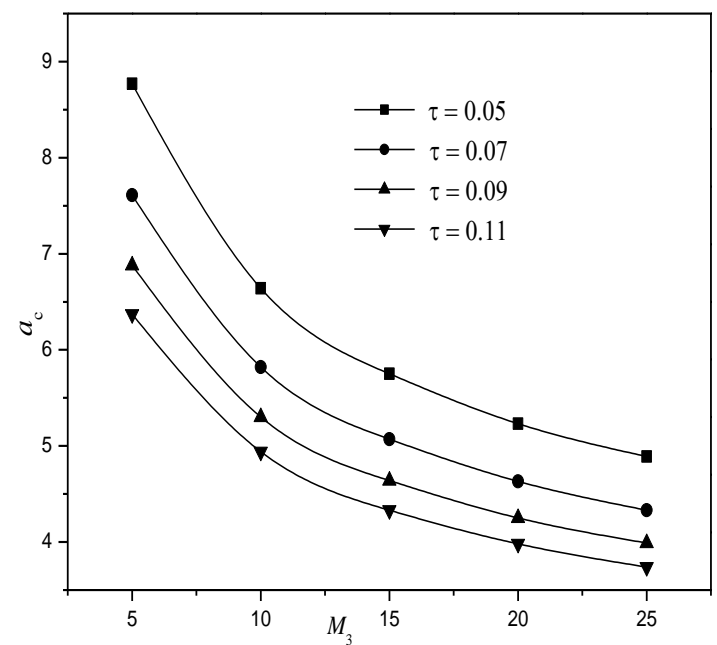

Fig. 5 (b). Variation of $a_{\mathrm{c}}$ versus $M_{3}$ for different $\tau$, $N_{1}=0.2, N_{3}{ }^{\prime}=2, N_{5}{ }^{\prime}=0.2$ and $R_{\mathrm{S}}=-500$.

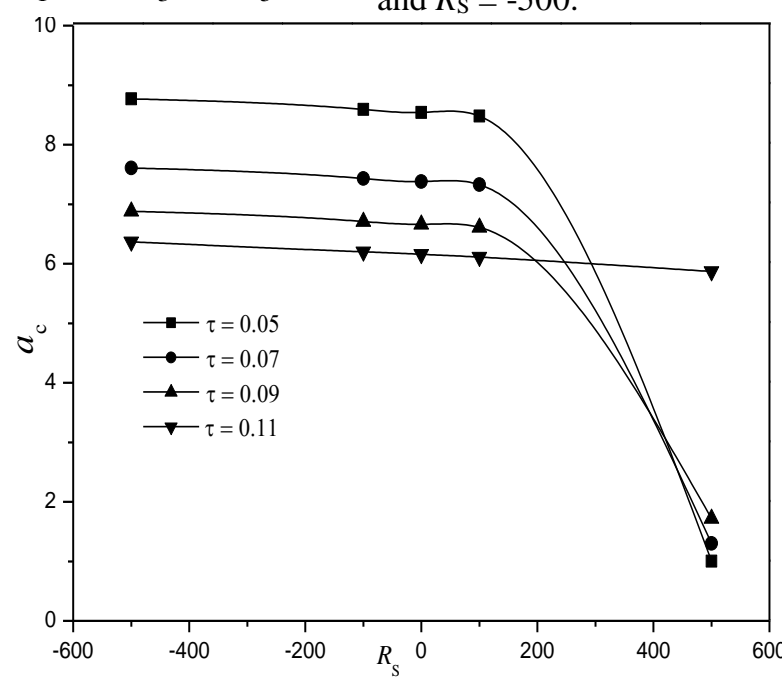

Fig. 6 (b). Variation of $a_{\mathrm{c}}$ versus $R_{\mathrm{S}}$ for different $\tau, N_{1}=0.2, N_{3}{ }^{\prime}=2, N_{5}{ }^{\prime}=0.2$ and $M_{3}=5$. 


\section{ACKNOWLEDGEMNT}

The author S. Seyalmurugan is grateful to Dr. M. Mohana Krishnnan, Principal, Jayagovind Harigopal Agarwal Agarsen College, Madhavaram, Chennai for his constant encouragement and thank to Dr.A. R. Vijayalakshmi, Department of Applied Mathematics, Sri Venkateswara College of Engineering, Sriperambudur, Chennai, for her support.

\section{REFERENCES}

1. R. E. ROSENSWEIG, Ferrohydrodynamics, Cambridge University Press, Cambridge, 1985.

2. S. CHANDRASEKHAR, Hydrodynamics and Hydromagnetic stability, Oxford University Press, London, 1961.

3. B. A. FINLAYSON, Convective instability of ferromagnetic fluids, International Journal of Fluid Mechanics, 40, pp.753-767, 1970.

4. G. VAIDYANATHAN, R.SEKAR, R. BALASUBRAMANIAN, Ferroconvective instability of fluids saturating a porous medium, International Journal of Engineering Science, 29, pp.1259-1267, 1991.

5. D. P. LALAS, S. CARMI, Thermoconvective stability of ferrofluids, Physics Fluids, 14, pp.436437, 1971.

C. ERINGEN, Theory of micropolar fluids, J. Math. Mech., 16, pp.1-18, 1966.

C. ERINGEN, Theory of thermomicrofluids, Journal of Mathematical Analysis and Applications, 38, pp.480-496, 1972.

6. T. ARIMAN, M. A. TURK, N. D. SYLVESTER, Applications of microcontinuum fluid mechanics, International Journal Engineering Science, 12, pp.273-293, 1974.

C. ERINGEN, Microcontinum fields theories, II: Fluent Media, Springer, New York, 2001.

7. G. AHMADI, Stability of micropolar fluid layer heated from below, International Journal Engineering Science, 14, pp.81-89, 1976.

PÉREZ-GARCIA, J. M. RUBI, On the possibility of overstable motions of micropolar fluids heated from below, International Journal Engineering Science, 20, pp.873 - 878, 1982.

8. Y. NARASIMMA MURTY, Analysis of non-uniform temperature profiles on Bénard convection in micropolar fluids, Applied Mathematics and Computation, 134, pp.473 - 486, 2003.

9. G. VAIDYANATHAN, R. SEKAR, A. RAMANATHAN, Ferro thermohaline convection, Journal of Magnetism and Magnetic Materials, 176, pp.321-330, 1997.

10. G. VAIDYANATHAN, R. SEKAR, A. RAMANATHAN, Ferro thermohaline convection in a porous medium, Journal of Magnetism and Magnetic Materials, 149, pp.137-142, 1995.

11. SUNIL, C. PRAGASH, P. K. BHARTI, Double diffusive convection in a micropolar ferromagnetic fluid, Applied Mathematics and Computation, 189, pp.1648-1661, 2007.

12. G. VAIDYANATHAN, R. SEKAR, R. HEMALATHA, R. VASANTHAKUMARI, S. SENTHILNATHAN, Soret-driven ferro thermohaline convection, Journal of Magnetism and Magnetic Materials, 288, pp.460-469, 2005.

13. R. SEKAR, G. VAIDYANATHAN, R. HEMALATHA, S. SENTHILNATHAN, Effect of sparse distribution pores in a Soret-driven ferro thermohaline convection, Journal of Magnetism and Magnetic Materials, 302, pp.20-28, 2006.

14. R. SEKAR, D. MURUGAN, K. RAJU, Stability analysis of thermohaline convection in ferromagnetic fluid in densely packed porous medium with Soret effect, World Journal of Engineering 10(5), 439-447, 2013.

15. REENA, U. S. RANA, Thermosolutal convection of micropolar rotating fluids saturated porous medium, IJE Transaction A: Basics 22, pp. 379-404, 2009.

16. S. CHAND, Linear stability of triple-diffusive convection in micropolar ferromagnetic fluid saturating porous medium, Applied Mathematics and Mechanics, 34, pp. 309-326, 2013.

C. ERINGEN, Simple microfluids, International Journal of Engineering Science, 2, 205-217, 1964. 